\title{
Mutagenic effects of gold nanoparticles induce aberrant phenotypes in Drosophila melanogaster
}

\author{
Giuseppe Vecchio, $\mathrm{PhD}^{\mathrm{a}, 1}$, Antonio Galeone, $\mathrm{MSc}^{\mathrm{a}, 1}$, Virgilio Brunetti, $\mathrm{PhD}^{\mathrm{a}}$, \\ Gabriele Maiorano, $\mathrm{PhD}^{\mathrm{a}}$, Loris Rizzello, $\mathrm{MSc}^{\mathrm{a}}$, Stefania Sabella, $\mathrm{PhD}^{\mathrm{a}}$, \\ Roberto Cingolani, $\mathrm{PhD}^{\mathrm{b}}$, Pier P. Pompa, $\mathrm{PhD}^{\mathrm{a}, *}$ \\ a Italian Institute of Technology, Center for Bio-Molecular Nanotechnologies @UniLe, Arnesano (Lecce), Italy \\ ${ }^{\mathrm{b}}$ Italian Institute of Technology, Central Research Laboratories, Genova, Italy \\ Received 17 October 2011; accepted 3 November 2011
}

\begin{abstract}
The peculiar physical/chemical characteristics of engineered nanomaterials have led to a rapid increase of nanotechnology-based applications in many fields. However, before exploiting their huge and wide potential, it is necessary to assess their effects upon interaction with living systems. In this context, the screening of nanomaterials to evaluate their possible toxicity and understand the underlying mechanisms currently represents a crucial opportunity to prevent severe harmful effects in the next future. In this work we show the in vivo toxicity of gold nanoparticles (Au NPs) in Drosophila melanogaster, highlighting significant genotoxic effects and, thus, revealing an unsettling aspect of the long-term outcome of the exposure to this nanomaterial. After the treatment with Au NPs, we observed dramatic phenotypic modifications in the subsequent generations of Drosophila, demonstrating their capability to induce mutagenic effects that may be transmitted to the descendants. Noteworthy, we were able to obtain the first nanomaterial-mutated organism, named NM-mut. Although these results sound alarming, they underline the importance of systematic and reliable toxicology characterizations of nanomaterials and the necessity of significant efforts by the nanoscience community in designing and testing suitable nanoscale surface engineering/coating to develop biocompatible nanomaterials with no hazardous effects for human health and environment.
\end{abstract}

From the Clinical Editor: While the clinical application of nanomedicine is still in its infancy, the rapid evolution of this field will undoubtedly result in a growing number of clinical trials and eventually in human applications. The interactions of nanoparticles with living organisms determine their toxicity and long-term safety, which must be properly understood prior to large-scale applications are considered. The paper by Dr. Pompa's team is the first ever demonstration of mutagenesis resulting in clearly observable phenotypic alterations and the generation of nano-mutants as a result of exposure to citrate-surfaced gold nanoparticles in drosophila. These groundbreaking results are alarming, but represent a true milestone in nanomedicine and serve as a reminder and warning about the critical importance of "safety first" in biomedical science.

(C) 2012 Elsevier Inc. All rights reserved.

Key words: Nanotoxicology; Gold nanoparticles; Drosophila melanogaster; Genotoxicity

Among hazardous agents, such as toxic chemicals and radiation, those eliciting genotoxic effects are typically classified as the most dangerous to human health and environment, because they induce DNA damage with consequent carcinogenic and/or mutagenic effects observable after long time periods or even in the following generations. ${ }^{1}$ For this reason, systematic

The authors declare that they have no conflicts of interest.

*Corresponding author: Center for Bio-Molecular Nanotechnologies@ UniLe, Italian Institute of Technology (IIT), Via Barsanti - 73010 Arnesano (Lecce), Italy.

E-mail address: pierpaolo.pompa@iit.it (P.P. Pompa).

${ }^{1}$ These authors contributed equally to this work. testing of all new materials and composites to assess possible genotoxic risk is of paramount importance. In this context, we are currently coming across a new class of materials that require an urgent evaluation of their potential harmful effects, i.e., nanomaterials. Actually, due to their increasing use in several technological fields $s^{2,3}$ and the consequent higher probability of their interaction with humans and the environment, ${ }^{4-6}$ a significant number of studies to assess their toxicity has been recently produced. ${ }^{6,7}$ In particular, many investigations have demonstrated both in vitro and in vivo that some nanomaterials exhibit clear cytotoxic ${ }^{8}$ and, sometimes, even genotoxic effects, ${ }^{9}$ thus raising novel and serious concerns. Moreover, to further worsen the situation is the large variability of the physical/ 
chemical properties of nanomaterials (size, shape, surface chemistry and charge, dispersion status, suspending medium, etc.), which significantly affects their toxic behavior. ${ }^{10-12}$ Hence, a reliable assessment of the different toxicological aspects of such materials, along with the identification of the key parameters responsible for the toxic effects, is essential. In this work, we analyzed in vivo the long-term genotoxic and mutagenic effects of citrate-capped $15 \mathrm{~nm} \mathrm{Au} \mathrm{NPs,} \mathrm{exploiting}$ the model organism Drosophila melanogaster. After the treatment with $\mathrm{Au}$ NPs, we observed significant phenotypic modifications in the subsequent generations of the organism, demonstrating their capability to induce mutagenic effects that may be transmitted to the progeny. Noteworthy, we were able to isolate and maintain one aberrant strain, named "nanomaterialmutated" (NM-mut), which represents the first nanomaterialmutated organism to date.

\section{Methods}

\section{$A u N P S$}

In this study we used highly monodispersed and stable 15-nm citrate-capped $\mathrm{Au}$ NPs synthesized and characterized in our laboratory, as previously described. ${ }^{11,13}$

\section{Drosophila melanogaster strain and culture conditions}

The flies and larvae of wild-type Drosophila melanogaster (Oregon R+, Bloomington Drosophila Stock Center, Bloomington, Indiana) were cultured at $24^{\circ} \pm 1^{\circ} \mathrm{C}$ on standard Drosophila sterile food containing agar, corn meal, sugar, yeast, and nepagin (methyl-p-hydroxybenzoate, Sigma Aldrich, St. Louis, Missouri). The use of Drosophila for these experiments was approved by the Italian Institute of Technology Scientific Committee for Animal Studies.

\section{Treatment schedule}

$\mathrm{Au}$ NPs were formulated in the diet at the final concentration of $100 \mathrm{pM}$ (namely, $2.05 \times 10^{-3} \mu \mathrm{g} / \mu \mathrm{l}$ of $\mathrm{Au}$ ). Assuming that Drosophila typically eats $1.5 \mu \mathrm{l} /$ day of food, ${ }^{14}$ the dose of NPs administered to the flies correspond to $3 \mu \mathrm{g} / \mathrm{g}$ per day. The solution containing $\mathrm{Au}$ NPs was added to the food before solidification, mixed strongly, and finally poured into vials. Transmission electron miscroscopy (TEM) analyses showed that the Au NPs do not significantly aggregate after mixing with the Drosophila food, maintaining a good degree of monodispersity (Figure S21). With the same modality, we prepared food with the $\mathrm{Au}$ NPs supernatant (SN), obtained by centrifugation of the $\mathrm{Au}$ NP solution at 13,400 rpm for 30 minutes. This preparation was used to exclude the presence of toxic compounds in the solution containing Au NPs.

For all the experiments the flies were treated for an entire life-cycle (eggs-to-eggs). In particular, groups of 10 flies were transferred in vials containing normal, SN, and Au NPs treated food and, after eggs deposition, the flies were removed. The virgin male and female flies, emerging from the control, the $\mathrm{SN}-$, and the Au NP-treated food (generation F0), were transferred in new vials (containing the respective food), and the adults were removed again after the eggs deposition. Finally, the flies emerging from the control, SN-, and Au NPtreated food (F1 generation) were immediately transferred in fresh normal food and used for all the various experiments reported in this article.

\section{Analysis of reproductive performance}

The virgin male and female flies of the F0 generation, emerging from the control, the SN-, and 15-nm Au NP-treated food, were pair-mated in vials having normal Drosophila food. In particular, ten couples of flies were divided in ten groups in three independent experiments for each treatment. Flies were transferred into fresh vials every day for the next 20 days, and the number of eggs laid during the period was scored. The total number of flies produced for 20 days by each pair was counted.

\section{Haemocytes collection}

D. melanogaster haemocytes were collected according to the methods of Irving et al ${ }^{15}$ with minor modifications. Briefly, chilled $96 \pm 2$-hour-old larvae were removed from food media, washed in water, sterilized in $5 \%$ bleach, and dried. The cuticle from 10 larvae was then disrupted with two fine forceps. The haemolymph and the circulating haemocytes were then collected in cold phosphate buffered saline (PBS) solution. The number of hemocytes per larvae typically ranged from 1000 to 1500 .

\section{TUNEL assay}

For Tunel assay, the cold solution of PBS containing the hemocytes was placed on a polylysine slide to allow electrostatic adhesion of cells. Subsequently, the sample was fixed in $4 \%$ paraformaldehyde in PBS for 15 minutes. After washing in PBST (PBS, 0.1\% Tween 20), the samples were permeabilized in a solution of PBS $0.3 \%$ Triton X 100 for 15 minutes. Hemocytes were then processed by Click-iT TUNEL Alexa Fluor647 Imaging Assay (Invitrogen, Eugene, Oregon), according to the manufacturer's instructions. Hemocytes were equilibrated for 10 minutes in TdT TUNEL buffer and then added to TdT reaction cocktail, containing TdT enzyme and a modified dUTP, and incubated for 60 minutes at $37^{\circ} \mathrm{C}$. After incubation, hemocytes were washed twice with $3 \%$ BSA in PBS for 2 minutes each. Subsequently, samples were incubated with Click-iT reaction cocktail for 30 minutes at room temperature $\left(20^{\circ}-22^{\circ} \mathrm{C}\right)$ in a dark environment. Then, the Click-iT reaction cocktail was removed and tissue was washed with 3\% BSA in PBS for 5 minutes, and a solution of $1 \mathrm{X}$ Hoechst 33342 was added for 15 minutes at room temperature. These samples were then characterized by confocal microscopy (Leica TCS-SP5 AOBS Leica Microsystems, Wetzlar, Germany). Quantitative analyses of TUNEL-positive nuclei were carried out by examining different hemocytes samples (20 different microscopic fields each) from three independent experiments.

\section{Annexin V/PI assay}

The analysis of apoptosis/necrosis on hemocytes was carried out with an Alexa Fluor 488 Annexin V/Dead Cell Apoptosis Kit (Invitrogen), according to the manufacturer's instructions. Briefly, hemocytes from 10 larvae were resuspended in $1 \times$ 
binding buffer in the range from 1000 to 1500 hemocytes per larva. Then, a solution containing propidium iodide (PI) and Alexa Fluor 488 annexin $\mathrm{V}$ was added to the hemocytes. Cells were gently vortexed and incubated for 15 minutes at room temperature in the dark. Finally, four-fold volume $1 \times$ binding buffer was added to each tube, and the samples were analyzed by confocal microscopy (Leica TCS-SP5 AOBS). Quantitative analyses of Annexin V/PI-positive cells were carried out by examining different hemocytes (20 different microscopic fields each) from three independent experiments.

\section{Expression analysis of p53 gene}

Third instar larvae extracts were prepared by homogenizing larvae in groups of ten in cold solution of RNAlater (Sigma Aldrich). Next, p53 mRNA expression levels were examined by performing real-time quantitative reverse transcription polymerase chain reaction (PCR) in larvae nurtured with Au NP-treated food and larvae nurtured with normal food. Total RNA was isolated from flies using TRI-reagent (Sigma Aldrich), the amount of RNA in each sample was determined by Nanodrop, and RNA quality was analyzed using agarose gel electrophoresis (1.2\%). Real-time qPCR was performed using direct RNAs in one-step reaction in an ABI 7500 thermocycler (Applied Biosystems, Carlsbad, California). For each gene, we used $1 \mu \mathrm{l}$ of $0.5 \mu \mathrm{g} / \mu \mathrm{l}$ of RNA solution mixed with $10 \mu \mathrm{l}$ of $10 \times$ Express Syber Green qPCR SuperMix premixed with ROX (Invitrogen), $2 \mu \mathrm{l}$ of 4 $\mu \mathrm{M}$ gene specific primers mix, $0.5 \mu \mathrm{l}$ of Express SuperScript Mix for one-Step Syber GreenER (Invitrogen) and $6.5 \mu \mathrm{l}$ of DEPC-treated water. Reaction conditions for all genes were: $50^{\circ} \mathrm{C}$ for 5 minutes to perform cDNA synthesis and then immediately followed by PCR quantification program, repeated 40 times $\left(15 \mathrm{~s}\right.$ at $95^{\circ} \mathrm{C}, 1$ minute at $\left.60^{\circ} \mathrm{C}\right)$. This program was followed by a melting curve program $\left(60^{\circ}-99^{\circ} \mathrm{C}\right.$ with a heating rate of $0.1^{\circ} \mathrm{C} / \mathrm{s}$ and continuous fluorescence measurements). Relative expression was calculated from cycle threshold values ( $\Delta \Delta \mathrm{Ct}$ method) using the RpL32 (ribosomal protein L32) expression as internal control for each sample.

The primers used in real time qPCR analysis were designed by online Primer-BLAST software of NCBI. In particular, we used Rp132 primer (forward: CGA GTT GAA CTG CCT TCA AGA TGA CCA and reverse: CCG ACT GGT GGC GGA TGA AGT G) that amplify the target sequence NM_001144656.1, and p53 primer (forward: TGC GGA CAC AAA TCG CAA CTG CT and reverse: ACG ACG CGG ACT TGT GAA GAC TC) that amplify the target sequences NM_001170223.1; NM_206544.2. All the target sequences are reported as NCBI accession number.

\section{Phenotypic observations}

The phenotype of flies grown on normal, SN-, and Au NPtreated food was observed using a stereomicroscope (Leica S8 APO, Leica Microsystems). In particular, the phenotypes of flies emerging from F0 (generation subjected to the treatment eggs-toeggs), F1 (first offspring generation), and F2 (second generation) were analyzed. Among all the various observed phenotypes, some types were collected and crossed with wild-type flies to assess the presence of gene mutations by propagation of the mutant phenotype in the progeny. In these experiments, we observed 2500 flies emerged from each treatment in the F0; among these, 1000 flies were randomly selected and separated in groups of 50 flies in 20 vials to obtain the F1 generation. In the F1 we analyzed the phenotype of approximately 10,000 flies per treatment, and also in this case 50 randomly selected flies were separated in 40 vials to obtain the F2 generation. Finally, we observed the phenotype of approximately 20,000 flies in F2.

\section{Results}

To study the long-term effects of Au NPs, we employed Drosophila melanogaster, a superb model for genetic and toxicological investigations, which has been widely used for the assessment of genotoxicity of various chemicals. ${ }^{16,17}$ Drosophila provides some advantages over higher organisms, being particularly suitable for massive screening studies, avoiding significant ethical issues, and allowing for the monitoring of the long-term effects induced by specific agents in several generations of the progeny, thanks to its short lifespan. On the other side, the assessment of the possible harmful effects of $\mathrm{Au}$ NPs is very important because they are widely employed in a broad range of applications. ${ }^{18-20}$ Notably, increasing evidence shows that nanoscale gold may induce toxic effects both in vitro and in vivo, especially in the absence of stable coatings or surface modifications. ${ }^{21-23}$ In addition, it has been recently demonstrated that Au NPs can induce remarkable variations in the genome-wide expression profile ${ }^{24}$ and genotoxic effects in vitro, ${ }^{25}$ though the underlying mechanisms are not yet clear. Currently, a mechanism of indirect DNA damage mediated by increased levels of reactive oxygen species (ROS) seems to be the most plausible hypothesis. ${ }^{9,22,25-28}$ These evidences are also corroborated by in vivo studies, in which Au NPs have been shown to induce apoptotic events in the liver of mice, ${ }^{29}$ or DNA damage in the gastro-intestinal tissue of $D$. melanogaster. ${ }^{13,30}$

As a first step, we analyzed different aspects of the reproductive performance of Drosophila upon ingestion of 15 $\mathrm{nm}$ Au NPs (3 $\mu \mathrm{g} / \mathrm{g}$ per day) (for all the experimental procedures see Methods). In particular, we studied the fecundity and fertility of the treated organisms by monitoring the number of eggs laid daily and the fraction of emerged flies. Typically, in the period ranging from the 5 th to the 25 th day after the pair mating, it is known that Drosophila populations reach the fecundity peak, keeping it constant for the entire period. ${ }^{31}$ Experimental results are shown in Figure 1, $A$ and highlight the significant difference in the behavior of treated flies with respect to the controls. The number of eggs laid daily by the organisms treated with Au NPs is significantly lower than that of the controls (CTRL and SN), which conversely show comparable values. In particular, the fecundity peak of treated flies is lower (approximately $60 \%$ of the control) and delayed, and maintains its maximum period for only 7 days (from the 12th to the 18th day), unlike control organisms in which the deposition rate is nearly constant for all 20 days, in close agreement with literature data. ${ }^{31}$ By analyzing the total number of eggs laid during the entire fecundity period (Figure 1, B), the harmful effects of $15 \mathrm{~nm}$ Au NPs are even more evident, revealing that the eggs deposited by treated organisms are only $46 \%$ of the 

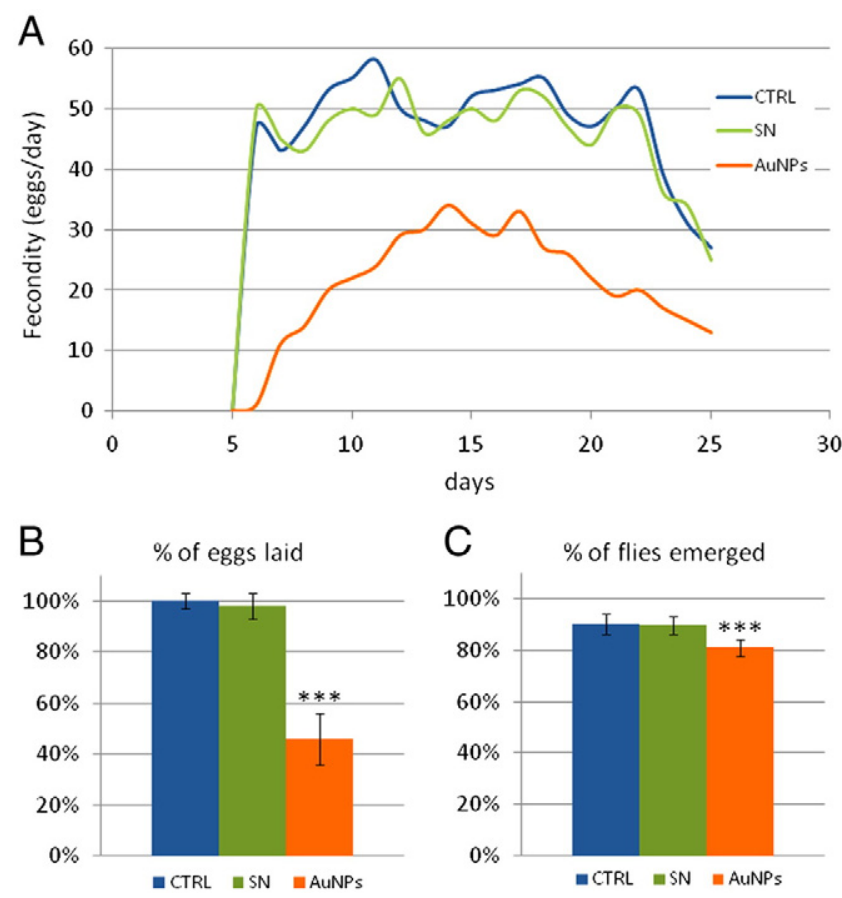

Figure 1. Analysis of the fertility performance of Drosophila upon treatment with $15 \mathrm{~nm}$ citrate capped Au NPs (3 $\mu \mathrm{g} / \mathrm{g}$ per day). (A) Number of eggs laid daily by Drosophila; (B) Total number of eggs deposited by the fly during the fecundity period (control normalized to $100 \%$ ); (C) Percentage of eclosed flies. Data are reported as mean $\pm \mathrm{SD}$ from three independent experiments. ${ }^{* * *} P<0.01$ (One-way ANOVA statistical analysis).

values observed for the controls. Moreover, there is also a slight decrease in the number of adult individuals developed in the treated samples (Figure 1,C). Beyond the physiological 10\% loss present in the control, ${ }^{32}$ an additional decrease of approximately $10 \%$ was detected in the Au NP-treated flies. The reduction of the eclosed flies can be attributed to a combination of development disorders induced by the toxicity of Au NPs with the occurrence of grave or lethal genetic mutations (see also below). Overall, these data indicate a strong perturbation of the health status of the organisms exposed to the NPs, specifically highlighting the presence of a generalized stress that, in turn, negatively affects the fertility performance. ${ }^{33}$

We further examined the toxicity mechanisms of Au NPs by investigating whether they exert adverse effects on the genome of Drosophila, as DNA damage by nanoscale gold has been recently reported in vitro, ${ }^{25}$ in ex vivo model systems, ${ }^{34}$ and in vivo. $^{13,30}$ To this purpose, we focused our studies on the circulating haemocytes of the hemolymph of Drosophila, which represent a widely recognized model to test the genotoxicity of chemical compounds. ${ }^{35}$ Moreover, since Drosophila hemocytes are analogous to the lymphocytes present in the blood of mammalians and execute the same function, ${ }^{15}$ the results of these experiments might be relevant also for higher organisms. In particular, we evaluated the presence of DNA damage in the hemocytes by TUNEL assay and the presence of apoptotic/ necrotic events by Annexin V/PI test. TUNEL data (Figure 2, $A$ ) revealed a significant presence of DNA fragmentation in treated
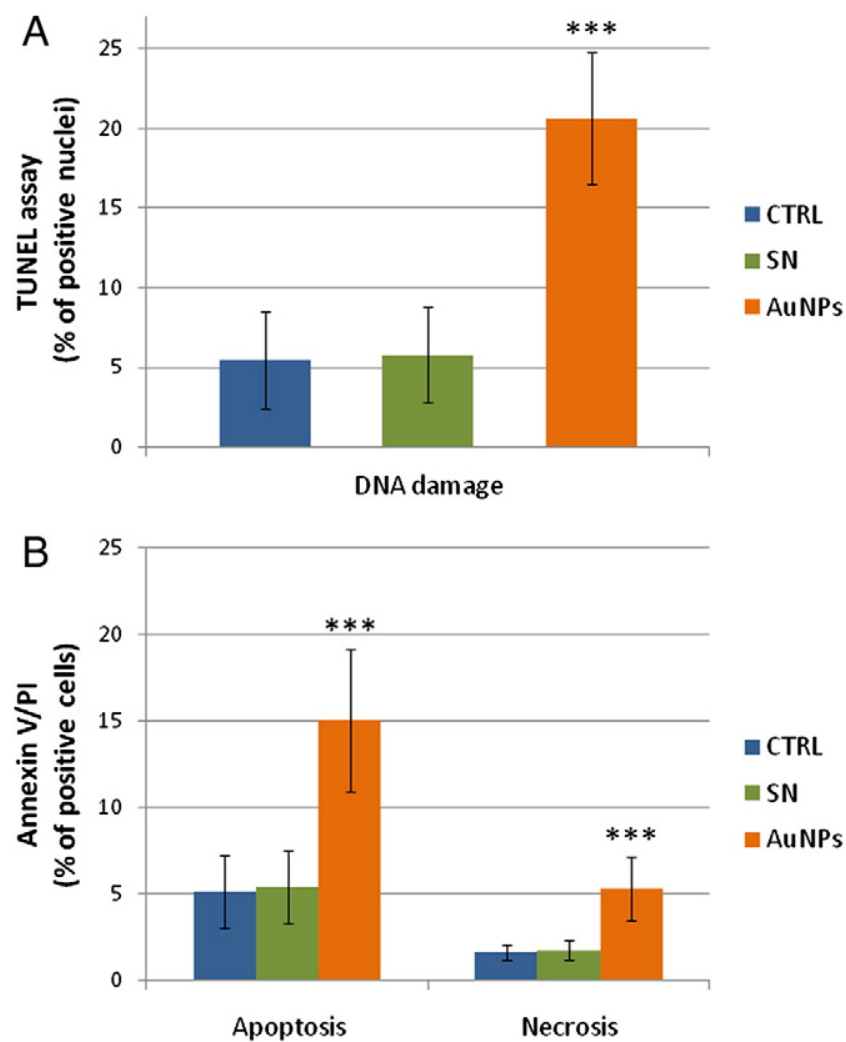

Figure 2. Genotoxic effects of Au NPs on Drosophila. (A) TUNEL assay and (B) apoptosis/necrosis assay performed on the circulating hemocytes of Drosophila. Data are reported as mean \pm SD from three independent experiments. ${ }^{* * *} P<0.01$ (One-way ANOVA statistical analysis).

organisms $(20.6 \pm 4.1 \%)$ with respect to the physiological level detected by the assay in control and SN flies $(5.5 \pm 3.0 \%$ and 5.8 $\pm 3.0 \%$, respectively). ${ }^{36,37}$ Such data were also confirmed by COMET assay $(17.1 \pm 2.9 \%$ of positive nuclei in treated flies compared to $3.7 \pm 1.4 \%$ and $3.5 \pm 1.6 \%$ of the control and $\mathrm{SN}$, respectively). Consistently, Annexin V/PI experiments (Figure 2, $B)$ showed that approximately $15 \%(15.0 \pm 4.1 \%)$ of hemocytes arising from $\mathrm{Au}$ NP-treated organisms present apoptotic processes, whereas approximatelv $5 \%(5.3 \pm 1.8 \%)$ of the cells undergo necrosis (or late apoptosis phase), unlike control and SN samples, which exhibit significantly lower values. It is noteworthy that these experimental results agree in indicating that $\mathrm{Au}$ NPs are able to induce clear genotoxicity. In particular, we found that the harmful effects of the NPs may cross the digestive system of Drosophila, reaching the hemolymph and causing severe DNA damage to the circulating hemocytes. $^{9,22,25-28}$ Although the molecular mechanisms of $\mathrm{Au}$ NPs toxicity are not yet well clarified, their interaction with sulfur groups of proteins likely plays an important role. In addition, aspecific biomolecules/Au NPs interactions (with consequent unfolding/inactivation/depletion of proteins and enzymes) as well as intracellular release of metal ions by uptaken NPs may lead to the alteration of downstream processes, including ROS overproduction.

The genotoxicity findings have been substantiated by molecular tests, in which we analyzed the mRNA expression 


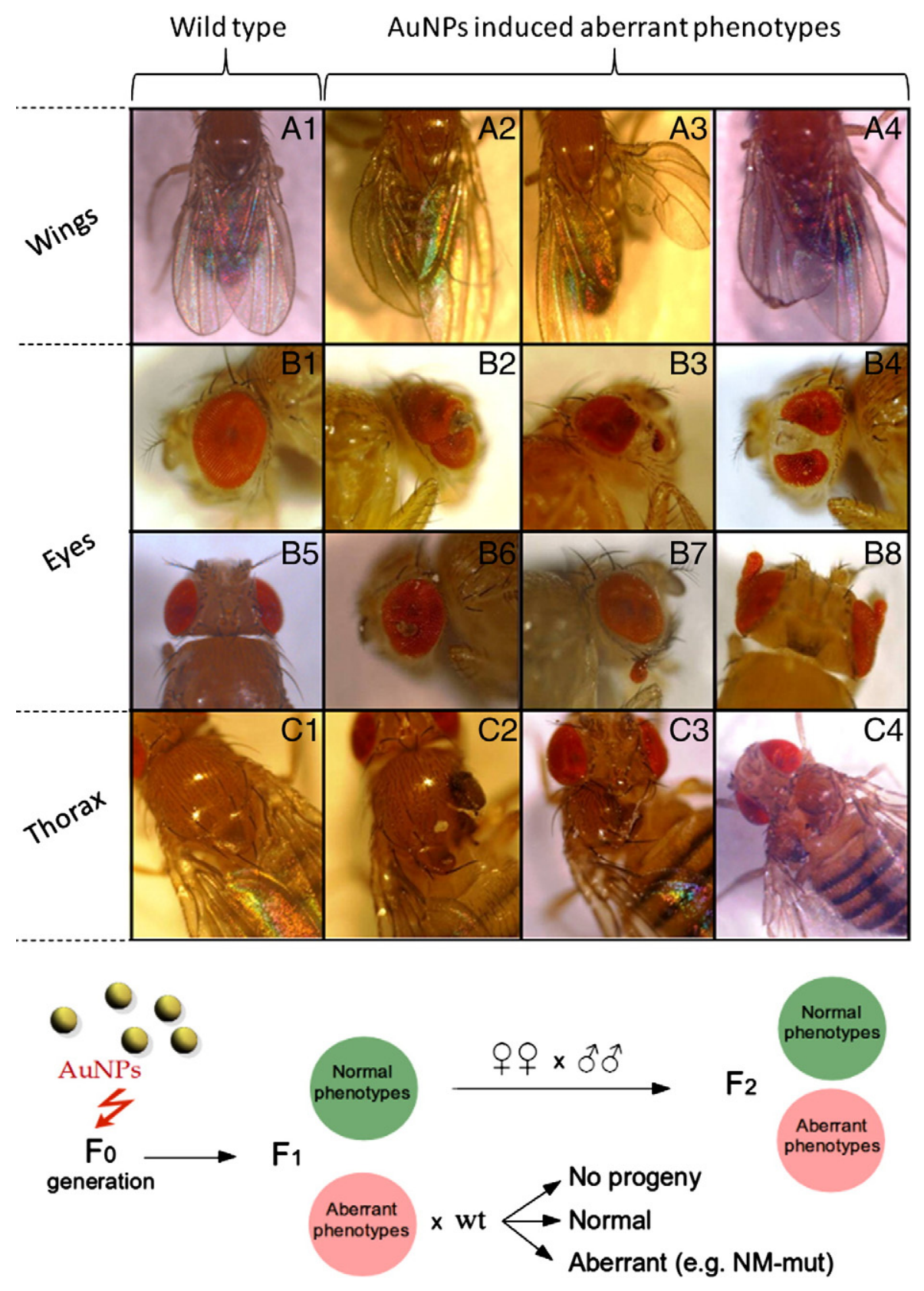

Figure 3. Representative images of mutant phenotypes observed in the progeny arising from organisms treated with Au NPs. In the first column, wild type flies are reported as reference. In the different rows, several body impairments are shown, such as deformations of wings (A series), eyes (B), and thorax (C). A scheme describing the flies mating used in these experiments is also reported.

level of p53 protein by real-time qPCR. P53 is a well known marker of the presence of DNA damage and, in treated organisms, it was found to be approximately threefold overexpressed $(2.99 \pm 0.15)$ with respect to the control. Typically, p53 is activated upon cellular stress, such as in the case of exposure to genotoxic agents, ionizing radiation, and hypoxia, ${ }^{38,39}$ playing a critical role in the maintenance of genomic integrity in multicellular organisms. ${ }^{40}$ In particular, the overexpression of p53 gene serves to prevent the formation of tumors and the propagation of mutations through the activation of the genes involved in the arrest of the cell cycle and the induction of apoptotic processes. ${ }^{38}$ Hence, the significant overexpression of p53 found in our Au NPs treatment is a reliable confirmation of the genotoxicity observed above, strongly suggesting the occurrence of considerable DNA damage.

The evidence of the remarkable genotoxicity found in Drosophila led us to focus our investigations on the possible longterm mutagenic effects induced by Au NPs. To this aim, we treated the flies with Au NPs for an entire life cycle (eggs-to-eggs) (F0 generation); afterwards, the newly eclosed flies of the subsequent generations (F1 and F2) were immediately transferred in new vials with fresh, untreated food, namely, the progeny was 
never exposed to the NPs. Flies were then subjected to a systematic screening of their phenotype. Although the individuals belonging to F0 exhibited the wild type phenotype, tough with a reduced viability, a detailed analysis of approximately 10,000 organisms of F1 led us to the discovery of the occurrence of abnormal phenotypes (beyond the possible presence of grave/ lethal mutations observed during the embryonic development, as discussed in Figure 1, C). In particular, we observed several flies characterized by an aberrant phenotype. Among those, some individuals revealed clear wing deformations (representative images are reported in Figure 3, $A$ series), whereas others presented strong malformations of the eyes; in particular, an impressive mutation resulting in a bisected eye with the anomalous presence of an extraneous structure in the middle is reported in Figure 3, B2. In the control samples, we observed only wild type flies.

All the abnormal phenotypes observed in F1 were crossed with wild type flies, and normal organisms were used for the production of the F2 offspring. In this latter case, we also observed surprising results. In fact, in the F2 generation we found some additional individuals with severely impaired body parts, despite the fact that they arose from parents that did not exhibit any morphological modification. Some representative examples of the mutant phenotypes observed in F2 are shown in Figure 3. We found malformed flies with an extraneous structure in the eyes similar to a protrusion with a bristle on its upper extremity (Figure 3, B6), flies presenting additional ectopic eyes (Figure 3, B7) or eyes protuberance covered with ommatidia (Figire 3, B8), and even severe malformations of the thorax with wings also malformed or completely absent (Figure 3, C2) or resulting in distortions of the longitudinal axis of the body (Figure 3, C3), or in the presence of two thoraxes, both deformed (Figure 3, C4).

On the other side, mutant individuals of F1 crossed with wild type flies were typically not able to generate progeny because of their very low viability and/or sterility. Among many attempts, we were able to maintain a mutated strain in only one case, namely the aberrant phenotype with malformed eyes reported in Figure 3, B2 (named "nanomaterial-mutated," or NM-mut).

\section{Discussion}

The NM-mut phenotype, despite its severe impairment, upon crossing with wild type organisms, was able to produce offspring, in turn able to reproduce further. In particular, the progeny obtained by NM-mut flies, pair-mated with normal organisms, typically exhibits approximately $50 \%$ of mutant phenotype, similar to that of the parents, and $50 \%$ with normal wild type morphology. Such percentages of mutants and normal individuals obtained by this pair mating indicates that this specific eye mutation is dominant. Moreover, by crossing these mutated individuals among them, we observed progeny with clear morphological defects of the eyes, such as large reduction of their size and/or presence of multiple ectopic eyes (up to six eyes). A couple of representative images are shown in Figure 3, $B 3-B 4$. Overall, such results unequivocally demonstrate that $15-$ $\mathrm{nm}$ Au NPs are able to induce genetic mutations in the germinal line of Drosophila that may be transmitted to the progeny. The aberrant strain NM-mut shown in Figure 3, B2 is currently maintained in our laboratory (where it continues to produce mutated offspring) and represents the first case of nanomaterialmutated organisms.

In the Supplementary Material, available online at http:// www.nanomedjournal.com, we report a wide series of additional images (Figures S1-S20) relative to several aberrant phenotypes observed during the experiments.

In conclusion, in this work we have shown that exposure to naked Au NPs may lead to severe consequences, highlighting, in general, how the lack of detailed knowledge about new materials might cause serious problems in the future. Despite the possibly alarming message of the presented results, with potential for dangerous drifts toward anxiety against nanotechnology, the aim of this paper is to highlight the strong opportunity that we have presently to understand the crucial necessity of massive and systematic screening of nanomaterials. The development of standardized nanotoxicology protocols and reliable in vivo models, along with the design and testing of suitable surface chemistry/coatings ${ }^{21,41}$ (for instance, the same 15-nm Au NPs coated with low molecular weight PEG were found to be not genotoxic), may lead to the efficient production of a wide class of safe nanomaterials-based products with no risks for humans or the environment.

\section{Appendix A. Supplementary data}

Supplementary to this article can be found online at doi: 10.1016/j.nano.2011.11.001.

\section{References}

1. MacGregor JT, Casciano D, Muller L. Strategies and testing methods for identifying mutagenic risks. Mutat Res 2000;455:3-20.

2. Mazzola L. Commercializing nanotechnology. Nat Biotechnol 2003;21: 1137-43.

3. Salata O. Applications of nanoparticles in biology and medicine. J Nanobiotechnol 2004;2:3.

4. Nel A, Xia T, Madler L, Li N. Toxic potential of materials at the nanolevel. Science 2006;311:622-7.

5. Service RF. Nanotoxicology. Nanotechnology grows up. Science 2004; 304:1732-4.

6. Oberdorster G, Oberdorster E, Oberdorster J. Nanotoxicology: an emerging discipline evolving from studies of ultrafine particles. Environ Health Perspect 2005;113:823-39.

7. Krug HF, Wick P. Nanotoxicology: an interdisciplinary challenge. Angew Chem Int Ed Engl 2011;50:1260-78.

8. Stone V, Donaldson K. Nanotoxicology - signs of stress. Nat Nanotechnol 2006;1:23-4.

9. Singh N, Manshian B, Jenkins GJ, Griffiths SM, Williams PM, Maffeis TG, et al. NanoGenotoxicology: the DNA damaging potential of engineered nanomaterials. Biomaterials 2009;30:3891-914.

10. Nel AE, Madler L, Velegol D, Xia T, Hoek EM, Somasundaran P, et al. Understanding biophysicochemical interactions at the nano-bio interface. Nat Mater 2009;8:543-57.

11. Maiorano G, Sabella S, Sorce B, Brunetti V, Malvindi MA, Cingolani R, et al. Effects of cell culture media on the dynamic formation of proteinnanoparticle complexes and influence on the cellular response. ACS Nano 2010;4:7481-91. 
12. Lundqvist M, Stigler J, Elia G, Lynch I, Cedervall T, Dawson KA. Nanoparticle size and surface properties determine the protein corona with possible implications for biological impacts. Proc Natl Acad Sci U S A 2008;105:14265-70.

13. Pompa PP, Vecchio G, Galeone A, Brunetti V, Sabella S, Maiorano G, et al. In Vivo toxicity assessment of gold nanoparticles in Drosophila melanogaster. Nano Res 2011;4:405-13.

14. Ja WW, Carvalho GB, Mak EM, de la rosa NN, Fang AY, Liong JC, et al. Prandiology of Drosophila and the CAFE assay. Proc Natl Acad Sci U S A 2007;104:8253-6.

15. Irving P, Ubeda JM, Doucet D, Troxler L, Lagueux M, Zachary D, et al. New insights into Drosophila larval haemocyte functions through genome-wide analysis. Cell Microbiol 2005;7:335-50.

16. Rubin GM, Lewis EB. A brief history of Drosophila's contributions to genome research. Science 2000;287:2216-8.

17. Würgler FE. Mutagenicity testing with Drosophila. Arch Toxicol 1980; 46:77-87.

18. Tan SJ, Campolongo MJ, Luo D, Cheng W. Building plasmonic nanostructures with DNA. Nat Nanotechnol 2011;6:268-76.

19. Giljohann DA, Seferos DS, Daniel WL, Massich MD, Patel PC, Mirkin CA. Gold nanoparticles for biology and medicine. Angew Chem Int Ed Engl 2010;49:3280-94.

20. Chin CD, Laksanasopin T, Cheung YK, Steinmiller D, Linder V, Parsa $\mathrm{H}$, et al. Microfluidics-based diagnostics of infectious diseases in the developing world. Nat Med 2011;17:1015-9.

21. Sabella S, Galeone A, Vecchio G, Cingolani R, Pompa PP. Au NPs are toxic in vitro and in vivo: a review. J Nanosci Lett 2011;1: $145-65$.

22. Li JJ, Hartono D, Ong CN, et al. Autophagy and oxidative stress associated with gold nanoparticles. Biomaterials 2010;31:5996-6003.

23. Balasubramanian SK, Jittiwat J, Manikandan J, Ong CN, Yu LE, Ong WY. Biodistribution of gold nanoparticles and gene expression changes in the liver and spleen after intravenous administration in rats. Biomaterials 2010;31:2034-42.

24. Massich MD, Giljohann DA, Schmucker AL, Patel PC, Mirkin CA. Cellular Response of Polyvalent Oligonucleotide-Gold Nanoparticle Conjugates. ACS Nano 2010;4:5641-6.

25. Li JJ, Zou L, Hartono D, Ong CN, Bay BH, Yung LY. Gold nanoparticles induce oxidative damage in lung fibroblasts in vitro. Adv Mater 2008;20:138-42.

26. Schaeublin NM, Braydich-Stolle LK, Schrand AM, Miller JM, Hutchison J, Schlager JJ, et al. Surface charge of gold nanoparticles mediates mechanism of toxicity. Nanoscale 2011;3:410-20.
27. Chompoosor A, Saha K, Ghosh PS, Macarthy DJ, Miranda OR, Zhu ZJ, et al. The role of surface functionality on acute cytotoxicity, ROS generation and DNA damage by cationic gold nanoparticles. Small 2010; 6:2246-9.

28. Gao W, Xu K, Ji L, Tang B. Effect of gold nanoparticles on glutathione depletion-induced hydrogen peroxide generation and apoptosis in HL7702 cells. Toxicol Lett 2011;205:86-95.

29. Cho WS, Cho M, Jeong J, Choi M, Cho HY, Han BS, et al. Acute toxicity and pharmacokinetics of $13 \mathrm{~nm}$-sized PEG-coated gold nanoparticles. Toxicol Appl Pharmacol 2009;236:16-24.

30. Pompa PP, Vecchio G, Galeone A, Brunetti V, Maiorano G, Sabella S, et al. Physical assessment of toxicology at nanoscale: nano dose-metrics and toxicity factor. Nanoscale 2011;3:2889-97.

31. Novoseltsev VN, Arking R, Carey JR, Novoseltseva JA, Yashin AI. Individual fecundity and senescence in Drosophila and medfly. J Gerontol A Biol Sci Med Sci 2005;60:953-62.

32. Key SCS, Reaves D, Turner F, Bang JJ. Impacts of Silver Nanoparticle ingestion on pigmentation and developmental progression in drosophila. Atlas J Biol 2011;1:52-61.

33. Nazir A, Mukhopadhyay I, Saxena DK, Chowdhuri DK. Chlorpyrifosinduced hsp70 expression and effect on reproductive performance in transgenic Drosophila melanogaster (hsp70-lacZ) Bg(9). Arch Environ Contam Toxicol 2001;41:443-9.

34. Skuridin SG, Dubinskaya VA, Rudoy VM, Dement'eva OV, Zakhidov ST, Marshak TL, et al. Effect of gold nanoparticles on DNA package in model systems. Dokl Biochem Biophys 2010;432:141-3.

35. Carmona ER, Creus A, Marcos R. Genotoxic effects of two nickelcompounds in somatic cells of Drosophila melanogaster. Mutat Res 2011;718:33-7.

36. Anderson KV, Matova N. Rel/NF-kappa B double mutants reveal that cellular immunity is central to Drosophila host defense. Proc Natl Acad Sci U S A 2006;103:16424-9.

37. Charroux B, Royet J. Elimination of plasmatocytes by targeted apoptosis reveals their role in multiple aspects of the Drosophila immune response. Proc Natl Acad Sci U S A 2009;106:9797-802.

38. May P, May E. Twenty years of p53 research: structural and functional aspects of the p53 protein. Oncogene 1999;18:7621-36.

39. Vousden KH. Activation of the $\mathrm{p} 53$ tumor suppressor protein. Biochim Biophys Acta 2002;1602:47-59.

40. Prives C, Hall PA. The p53 pathway. J Pathol 1999;187:112-26.

41. Verma A, Uzun O, Hu Y, Hu Y, Han HS, Watson N, et al. Surfacestructure-regulated cell-membrane penetration by monolayer-protected nanoparticles. Nat Mater 2008;7:588-95. 